Case Study

\title{
Cytomegalovirus Primary Infection in an Immunocompetent Female with Mononucleosis Features: A Review of Mononucleosis-Like
} Syndromes

Shohinee Sarma, MD MPH, Derek Little, MD, Tooba Ali, MBBS, Emily Jones, MD, Shariq Haider, MD FRCPC

DOI: $10.22374 /$ cjgim.v13i3.258

\section{About the Authors}

Shohinee Sarma and Derek Little are Internal Medicine PGY-2 at McMaster University in Hamilton, Ontario. Tooba Ali is an Adult Rheumatology PGY-4, Queen's University in Kingston, Ontario. Emily Jones is an Internal Medicine PGY-5, McMaster University in Hamilton, Ontario. Shariq Haider, is Professor, Division of Infectious Diseases, Department of Medicine, McMaster University in Hamilton, Ontario.

Submitted: November 12, 2017. Accepted: January 22, 2018. Published August 27, 2018.

\begin{abstract}
The clinical triad of fever, pharyngitis, and lymphadenopathy was first described in 1889 as "glandular fever" and later defined as infectious mononucleosis. We present a case report and review of mononucleosis-like syndromes in an immunocompetent patient. The review of common etiologies includes Epstein-Barr virus, acute human immunodeficiency virus, human herpesvirus 6, cytomegalovirus, and Toxoplasmosis gondii.
\end{abstract}

\section{RESUME}

La triade clinique de la fièvre, de la pharyngite et de la lymphadénopathie a été décrite pour la première fois en 1889 sous le nom de "fièvre glandulaire » et plus tard définie comme une mononucléose infectieuse. Nous présentons un rapport de cas et un examen des syndromes de type mononucléose chez un patient immunocompétent. L'examen des étiologies communes comprend le virus d'Epstein-Barr, le virus de l'immunodéficience humaine aiguë, l'herpèsvirus humain 6, le cytomégalovirus et la toxoplasmose gondii.

A 37-year-old, immunocompetent female was referred to the emergency department by her family physician with a three-week history of fever, pharyngitis, fatigue, night sweats, and abdominal pain. She was treated with a one-week course of clarithromycin without improvement by her family physician as an outpatient.

The patient was otherwise healthy. Past medical history was significant for endometriosis and infertility. She was pursuing clomiphene citrate inductions.

She was afebrile in the emergency department. Vital signs demonstrated sinus tachycardia at $100 \mathrm{bpm}$, respiratory rate 18 , oxygen saturation $98 \%$ on room air, blood pressure of $154 / 99$ $\mathrm{mmHg}$. Physical examination revealed hepatosplenomegaly, but no lymphadenopathy, rashes, or tender joints. The posterior pharynx was clear, and the remainder of her examination was unremarkable.

Previous laboratory investigations by her family physician noted lymphocytosis with smudge cells. However, investigations in hospital showed lymphocytosis (WBC $11.6 \times 10^{9} / \mathrm{L}$ and lymphocytes of $8.6 \times 10^{9} / \mathrm{L}$ ) and a normal peripheral smear. An inpatient hematology consult excluded hematological malignancy. Computed tomography of the abdomen confirmed hepatosplenomegaly with no lymphadenopathy. Her CD4/CD8 ratio was 0.2 in keeping with a viral infection. Bacterial cultures were negative. Serology for hepatitis B and C were negative. Human immunodeficiency 
virus (HIV) testing was not done in the absence of risk factors and a normal percent CD4 count. Epstein-Barr virus (EBV) monospot test was negative. Quantitative polymerase chain reaction (PCR) for cytomegalovirus (CMV) in blood samples was positive with a value of 965.25 units per $\mathrm{mL}$ using real-time PCR assay.

The patient was diagnosed with CMV viremia and treated with a two-week course of valganciclovir with resolution of symptoms. The decision to treat this patient was based on the persistence of symptoms over three weeks. Two-month follow-up revealed a healthy patient with a normal complete blood count. Hematology follow-up was arranged and outpatient abdominal

Table 1. Differential Diagnosis for Mononucleosis-like Illnesses

\begin{tabular}{|l|}
\hline Infectious \\
\hline Viral \\
\hline Cytomegalovirus \\
\hline Human herpes vírus-6 \\
\hline Human immunodeficiency vírus \\
\hline Adenovirus \\
\hline Enteroviruses \\
\hline Hepatitis vírus A \\
\hline Hepatitis vírus B \\
\hline Rubella vírus \\
\hline Bacterial \\
\hline Group A Beta-hemolytic streptococcus pyogenes \\
\hline Bartonella henselae \\
\hline Corynebacterium diptheriae \\
\hline Brucella spp. \\
\hline Francisella tularensis \\
\hline Borrelia spp. \\
\hline Mycobacterium tuberculosis \\
\hline Protozoal \\
\hline Toxoplasmosis gondii \\
\hline Malignancy \\
\hline Non-Hodgkin lymphoma \\
\hline Hodgkin lymphoma \\
\hline Inflammatory \\
\hline Systemic lupus erythematosis \\
\hline Sarcoidosis \\
\hline Medication reactions \\
\hline Carbamezapine \\
\hline Phenytoin \\
\hline Serum sickness hypersensitivity reaction \\
\hline
\end{tabular}

ultrasonography showed resolving hepatosplenomegaly. Repeat quantitative CMV PCR samples were negative.

\section{Review}

The clinical triad of fever, pharyngitis, and lymphadenopathy was first described in 1889 as "glandular fever" and later defined as infectious mononucleosis. ${ }^{1}$ A typical mononucleosis syndrome presents with acute febrile illness with more than $50 \%$ lymphocytes or monocytes, with at least $10 \%$ atypical lymphocytes. ${ }^{1}$ In an immunocompetent patient with mononucleosis-like symptoms, the differential diagnosis includes EBV, acute HIV, human herpesvirus 6 (HHV-6), cytomegalovirus CMV, Toxoplasmosis gondii and Group A beta-hemolytic streptococcus (GAS) (Table 1). We present a review of these etiologies and their clinical presentations.

\section{Epstein-Barr Virus}

EBV is a herpesvirus with a worldwide prevalence of $95 \%$ in adults. 3 Primary infection often occurs in children ages one to five and manifests as a non-specific viral illness. ${ }^{3}$ It commonly develops in adolescents and young adults with a peak in the second and third decades of life. ${ }^{2,3}$ Patients present with fever, pharyngitis, posterior cervical lymphadenopathy (bilateral, tender, and nonfixed), and malaise. Other findings include hepatosplenomegaly, jaundice, and palatal petechiae.,

Investigations demonstrate a lymphocytosis with atypical lymphocytes and mildly elevated liver enzymes in $50 \%$ of cases. ${ }^{2}$ Diagnoses can be confirmed with a monospot heterophile antibody test or EBV-specific antibody testing. A positive monospot test is highly specific but can be falsely negative in $25 \%$ of patients within the first week of symptom onset, $5-10 \%$ in the second week, and 5\% in the third week. Repeat testing or EBV-specific antibody testing is useful. ${ }^{2}$ IgG and IgM antibodies against viral capsid antigens and nuclear antigens are more specific. ${ }^{3}$

Treatment is supportive with adequate fluid intake, rest, and analgesia (non-steroidal anti-inflammatory drugs or acetaminophen). There is insufficient evidence to support the use of corticosteroids for symptom control. However, they may be considered for upper airway obstruction due to hypertrophy of lymphoid tissue, occurring in $1 \%$ of cases. ${ }^{3}$ There is no benefit to treatment with acyclovir or ranitidine. ${ }^{2}$ Other complications, although rare, include acute interstitial nephritis, autoimmune hemolytic anemia, myocarditis, cranial nerve palsies, encephalitis, meningitis, mononeuropathies, and thrombocytopenia. ${ }^{2}$ The risk of splenic rupture is $0.1 \%$ and contact sports should be avoided for a minimum of three to four weeks for those with associated splenomegaly. ${ }^{2}$

\section{Cytomegalovirus}

CMV is a member of the Herpesviridae family with a worldwide prevalence of $40-100 \%$ in adults. It is acquired early in life 
through congenital transmission. Primary CMV mononucleosis is uncommon in adults without close contact with children. ${ }^{4}$ Risk of transmission occurs with sexual contact or shedding in saliva, urine, or breast milk. ${ }^{4} \mathrm{CMV}$ is associated with severe primary illness and reactivation in immunocompromized hosts including transplant recipients and HIV-positive patients. ${ }^{4}$ Common clinical presentations in the immunocompromized host include pneumonitis, hepatitis, uveitis, retinitis, colitis, encephalitis, and graft rejection. ${ }^{5}$ Primary CMV infection in immunocompetent hosts is relatively scant in the medical literature and is usually asymptomatic. ${ }^{4,5}$

In $10 \%$ of immunocompetent cases, primary CMV infection presents as a mononucleosis syndrome. Symptoms common to both EBV and CMV include fever, malaise, sore throat, headache, and fatigue. However, CMV is less associated with tonsillitis, pharyngitis, and lymphadenopathy compared to EBV. ${ }^{4,6}$ Splenomegaly and lymphadenopathy occur less frequently in immunocompetent hosts. ${ }^{4}$ A 2008 systematic review of severe CMV disease in 290 immunocompetent patients revealed the gastrointestinal tract and central nervous system as the primary sites of infection. ${ }^{7}$ The prevalence of CMV pneumonia is as high as $50 \%$ in immunocompetent critically ill patients with ventilatorassociated pneumonia or acute respiratory distress syndrome. 5

Diagnostic testing includes CMV IgM and IgG serology for acute infections and reactivation. ${ }^{4}$ Quantitative or qualitative PCR is the gold standard for diagnosis. ${ }^{5}$ Presence of owl's eye viral inclusion bodies in histopathological specimens is highly specific. ${ }^{8}$

Treatment of immunocompetent patients has limited evidence. Antiviral drugs including ganciclovir, valganciclovir, and foscarnet demonstrate successful clinical outcomes. ${ }^{5}$ Since the disease is self-limiting, severity of symptoms, critical illness, and endorgan damage determine treatment decisions. ${ }^{7}$ The primary care provider should maintain a high index of suspicion for CMV in a heterophile-negative mononucleosis presentation. ${ }^{6}$

\section{Human Herpes Virus 6}

HHV-6, formerly described as human B-lymphotropic virus, was initially isolated in immunocompromized HIV patients. ${ }^{9}$ It is usually associated with roseola infantum in childhood. ${ }^{9}$ Primary infection in immunocompetent adults is rare, but reported as a mild, self-limiting mononucleosis-like syndrome. Symptoms include prolonged fever, myalgia, headaches, abdominal pain, and cervical lymphadenopathy. ${ }^{9} \mathrm{HHV}-6$ can be associated with encephalitis presenting with altered level of consciousness, seizures, psychosis, cerebellar ataxia, and neurologic deficits. ${ }^{10}$ The disease course can be severe and prolonged in immunocompromized hosts including solid organ and hematopoietic stem cell transplant patients. ${ }^{9}$

Since most people after 2 years of age are seropositive, paired IgM and IgG positive serology with a greater than four-fold increase is considered diagnostic. Quantitative PCR in serum, plasma, and cerebrospinal fluid has high sensitivity and specificity in primary infections. ${ }^{9,10}$

HHV-6 infections in immunocompetent patients are selflimiting and not generally treated. Antiviral agents such as foscarnet (HHV-6A and HHV-6B) and ganciclovir (HHV-6B) can be used in children, immunocompromized patients or those with severe disease. ${ }^{11}$

\section{Human Immunodeficiency Virus Type 1}

Acute infection with HIV virus type 1 (HIV-1) presents with a selflimiting mononucleosis-like syndrome lasting two to four weeks. ${ }^{12}$ Symptoms include fever, pharyngitis, myalgia, headache, rash, lymphadenopathy, oral and genital ulcerations, and gastrointestinal disorders. ${ }^{13}$ HIV is often a diagnosis that is missed in the acute phase.13 Approximately, 50\% of HIV patients worldwide are diagnosed in later stages of disease with CD4+ T-cell counts less than 350 cells $/ \mathrm{mm}^{3}$ or an AIDS-defining illness. Late detection decreases life expectancy, increases HIV transmission, and creates complexity in treatment and drug adherence. ${ }^{14}$

A British retrospective study of 1045 patients with glandular fever reported that $72.7 \%$ of cases were missed at the initial outpatient consultation. ${ }^{12}$ Retrospective testing from 563 patients with negative EBV heterophile tests showed that $1.2 \%$ of patients actually had primary HIV-1 infection. ${ }^{13}$ Another recent cohort study demonstrated that missed opportunities for HIV diagnosis often occurred despite healthcare access. ${ }^{14}$ The majority of missed diagnoses occurred in primary care centres (60\%) and in hospitalized settings including internal medicine, surgical, oncology, psychiatry, and dermatology departments. Qualitative data noted the primary reason for not testing was the perception that the patient did not have high risk factors. ${ }^{14}$ Late presentations included hematologic disorders, herpes zoster rash, severe unexplained dermatologic presentations, newly diagnosed Hepatitis $\mathrm{B}$ and $\mathrm{C}$ infection, unexplained weight loss, and infectious mononucleosis. ${ }^{14}$

Nucleic acid testing for HIV RNA provides the earliest detectable evidence. In the early symptomatic stage, enzymelinked immunosorbent assay (ELISA) testing can be negative, but HIV-1 RNA and plasma p24 antigen testing are used. ${ }^{13}$ Healthcare providers should perform ongoing risk factor assessments to allow for early diagnosis and antiretroviral treatment. ${ }^{13}$

\section{Toxoplasmosis Gondii}

Toxoplasmosis gondii is an obligate intracellular protozoan parasite that infects birds and mammals. Acute infection occurs after transmission of the parasite by the fecal-oral route from contaminated cat feces, consumption of undercooked or raw meat containing tissue cysts, or transplacentally. ${ }^{16}$ Reactivation of latent infection occurs primarily in immunocompromized patients. 
Acute infection in immunocompetent adults is largely asymptomatic with only $10 \%$ developing a self-limited and nonspecific illness. ${ }^{15}$ Symptoms include malaise, fever, and cervical or occipital lymphadenopathy (tender or non-tender, discrete, and firm). ${ }^{1,2}$ Illness may also manifest with fever, pharyngitis, myalgia, and atypical lymphocytosis. ${ }^{16}$ Rare complications include chorioretinitis, myocarditis, polymyositis, hepatitis, pneumonitis and encephalitis. ${ }^{15,16}$ Reactivation in immunocompromized patients can be life-threatening and most commonly manifests as toxoplasmic encephalitis with altered level of consciousness, focal neurological deficits, seizures, and other neurological symptoms. ${ }^{15}$

Diagnostic tests include serologic identification of antitoxoplasmosis IgM and IgG antibodies. IgG levels appear after 1-2 weeks, peak at 6-8 weeks, and then decline to a baseline detectable level for life. ${ }^{16}$ IgM levels rise after acute infection and remain elevated for up to 18 months. ${ }^{16}$ Acute infection in immunocompetent adults rarely requires treatment. Severe symptoms can be treated with a combination of pyrimethamine, sulfadiazine, and folinic acid for $4-6$ weeks. ${ }^{15}$

\section{Group A Beta-Hemolytic Streptococcus}

GAS accounts for approximately $5-15 \%$ of infectious acute pharyngitis, predominantly in those under 15 years old. Recognition of this pathogen is of importance due to the risk of developing rheumatic fever if left untreated. However, rates of rheumatic fever after GAS pharyngitis are rare. According to the Infection Disease Society of America, GAS is the only cause of acute pharyngitis which should be treated with antibiotics. ${ }^{17}$

Clinical presentation usually includes fever, sore throat, and odynophagia. Tonsillar exudates and tender cervical lymphadenopathy may occur. Unlike EBV, which involves the reticuloendothelial system, GAS typically lacks a systemic lymphocytosis or hepatosplenomegaly. There are multiple clinical prediction tools to diagnose GAS pharyngitis and guide appropriate antibiotic treatment.18 Unfortunately, they all lack specificity. ${ }^{19}$

Current guidelines recommend all suspected cases have throat swabs performed for rapid antigen testing, unless they have clear viral symptoms of coryza or rhinorrhea. ${ }^{17}$ Positive rapid antigen testing does not require confirmation with bacterial culture, nor should negative rapid antigen tests be confirmed with bacterial culture. Treatment of confirmed cases is with penicillin or a cephalosporin for a 10- day course.

Non-group A streptococci can also cause acute pharyngitis with a clinical presentation similar to GAS. ${ }^{20,21}$ However there is little evidence to support treating these infections. ${ }^{21}$

\section{Discussion}

In this case, the patient's hepatosplenomegaly and previous laboratory investigations showing smudge cells raised the suspicion for a hematologic malignancy. However, a hematology consult in hospital did not show any evidence of smudge cells with repeat blood work and outpatient follow-up demonstrated resolving hepatosplenomegaly with valgancyclovir treatment.

In immunocompetent patients presenting with symptoms of mononucleosis, the differential diagnosis should include other possibilities that fit a mononucleosis-like syndrome. CMV commonly affects young patients and is less associated with tonsillitis, pharyngitis, and lymphadenopathy. HHV-6 can present with headaches, encephalitis, and abdominal pain. Consideration of acute HIV mononucleosis should prompt early serologic testing. Toxoplasmosis is often associated with undercooked food or cat excrement, requiring anti-IgM antibody testing to distinguish from EBV. Although EBV infectious mononucleosis may be suspected, the general practitioner should consider a complete review of other infectious etiologies.

\section{Competing Interests}

All authors declare that they have no competing interests.

\section{Funding}

There was no funding source for this case report.

\section{Contributors}

All authors contributed to the case presentation, literature review, and final draft. All authors were involved in the editing process and approve the version submitted for publication and agree to be accountable for all aspects of the work in ensuring that questions related to the accuracy or integrity of any part of the work are appropriately investigated and resolved.

\section{Informed Consent}

Written informed consent was obtained from the patient described in the case report. A copy of the informed consent is available in the patient's chart if requested. The patient was advised that their name and initials are not included in the manuscript and we will endeavour to conceal their identity.

\section{References}

1. Hurt C, Tammaro D. Diagnostic evaluation of mononucleosis-like illnesses. Am J Med 2007;120,911e1-911e8.

2. Ebell MH. Epstein-Barr virus infectious mononucleosis. Am Fam Physician 2004;70:1279-87,1289-90.

3. Luzuriaga K, Sullivan JL. Infectious Mononucleosis. N Eng J Med 2010;362:1993--2000

4. Taylor GH. Cytomegalovirus. Am Fam Physician 2003;67(3):519-24.

5. Al-Omari A, Aljamaan F, Alhazzani W, Salih S, Arabi Y. Cytomegalovirus infection in immunocompetent critically ill adults: literature review. Ann Intensive Care 2016;6:110.

6. Klemola E, von Essen R, Henle G, Henle W. Infectious-mononucleosislike disease with negative heterophil agglutination test. clinical features in relation to Epstein-Barr virus and cytomegalovirus antibodies. J Infect Diseas 1970;121(6):608-14. 
7. Rafailidis P, Mourtzoukou E, Varbobitis I, Falagas M. Severe cytomegalovirus infection in apparently immunocompetent patients: a systematic review. Virol J 2008;5(1):47.

8. Mattes $\mathrm{F}$ et al. Histopathological detection of owl's eye inclusions is still specific for cytomegalovirus in the era of human herpesviruses 6 and 7. J Clin Pathol 2000;53(8):612-14.

9. Steeper TA, Horwitz CA, Ablashi DV, Salahuddin SZ, Saxinger C, Saltzman $\mathrm{R}$ et al. The spectrum of clinical and laboratory findings resulting from human herpesvirus-6 (HHV-6) in patients with mononucleosis-like illnesses not resulting from Epstein-Barr virus or cytomegalovirus. AJCP 1990;93(6):776-83.

10. Birnbaum T, Padovan CS, Sporer B, Rupprecht TA, Ausserer H, Jaeger G, et al. Severe meningoencephalitis caused by human herpesvirus 6 type B in an immunocompetent woman treated with ganciclovir. Clin Infect Dis 2005;40(6):887.

11. Crawford JR, Kadom N, Santi MR, Mariani B, Lavenstein BL. Human herpesvirus 6 rhomboencephalitis in immunocompetent children. J Child Neurol 2007;22(11):1260.

12. Hsu D, Ruf M, O’Shea S, Costelloe S, Peck J, Tong C. Diagnosing HIV infection in patients presenting with glandular fever-like illness in primary care: are we missing primary HIV infection? HIV Med 2012;14(1):60-63.
13. Rosenberg ES, Caliendo AM, Walker BD. Acute HIV Infection among Patients Tested for Mononucleosis. New Engl J of Med 1999;340(12):969.

14. Levy I et al. Missed opportunities for earlier diagnosis of HIV in patients who presented with advanced HIV disease: a retrospective cohort study. BMJ Open 2016;6(11):e012721.

15. Montoya JG, Liesenfeld O. Toxoplasmosis. Lancet 2004;363(9425):1965-76.

16. Gagne SS. Toxoplasmosis. Prim Care Update Ob/Gyns 2001;8(3):122-26.

17. Shulman ST, Bisno AL, Clegg HW, et al. Clinical Practice Guideline for the Diagnosis and Management of Group A Streptococcal Pharyngitis: 2012 Update by the Infectious Diseases Society of America Clin Infect Dis 2012;55(10):e86-e102.

18. Ebell MH, Smith MA, Barry HC, Ives K, Carey M. Does this patient have strep throat? JAMA 2000;284(22):2912-8.2.

19. Bisno AL, Peter GS, Kaplan EL. Diagnosis of strep throat in adults: are clinical criteria really good enough? Clin Infect Dis 2000;15;35(2):126-9.

20. Tiemstra J. Role of non-group a streptococci in acute pharyngitis. J Amer Board Fam Med 2009;22(6)663-69.

21. Thai TN, Dale AP, Ebell MH. Signs and symptoms of Group A versus NonGroup A strep throat: A meta-analysis. Fam Pract 2018 May 23;35(3):231-38. doi: $10.1093 /$ fampra/cmx072. 